\title{
Correction to: Motion planning for redundant multi-bodied planar kinematic snake robots
}

\author{
Omar Itani · Elie Shammas (1)
}

Published online: 26 July 2021

(C) Springer Nature B.V. 2021

Correction to: Nonlinear Dyn (2021) 104:3845-3860

https://doi.org/10.1007/s11071-021-06515-y

The online article presented the figures in black and white. The colored figures are presented here.

The online version of the original article can be found under https://doi.org/10.1007/s11071-021-06515-y.

O. Itani · E. Shammas $(\varangle)$

Vision and Robotics Lab, Department of Mechanical Engineering, American University of Beirut, Beirut, Lebanon

e-mail: es34@aub.edu.lb

O. Itani

e-mail: oji00@mail.aub.edu

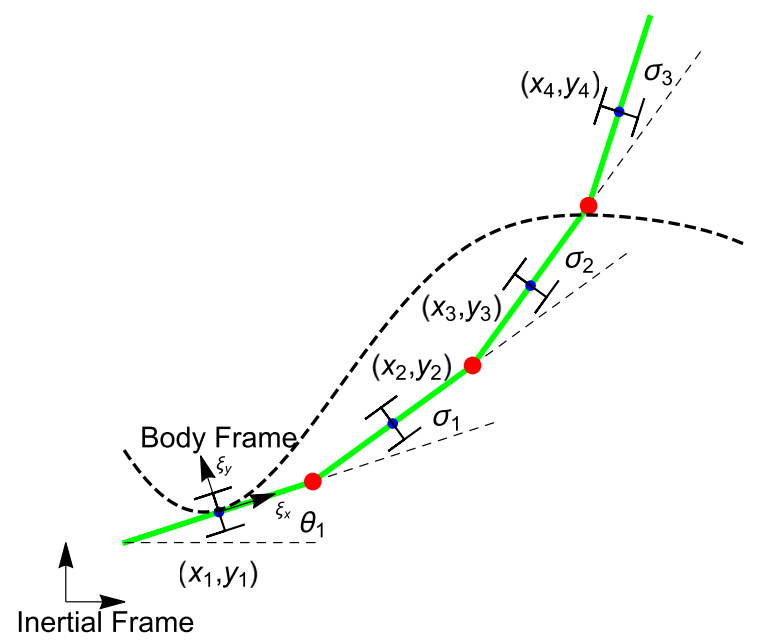

Fig. 1 Four-link planar nonholonomic snake robot

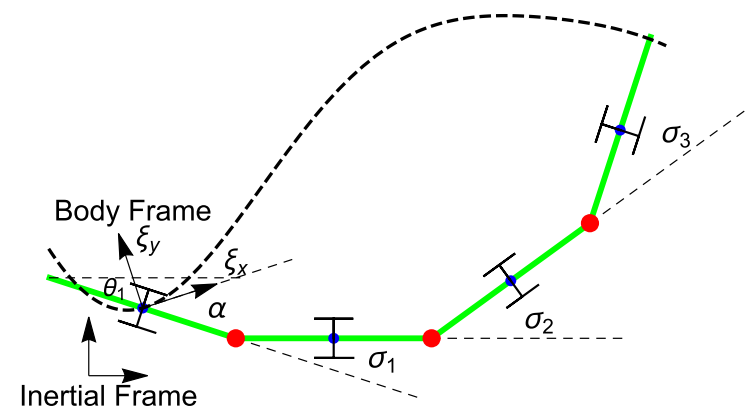

Fig. 2 Four-link snake with a variable body frame orientation 


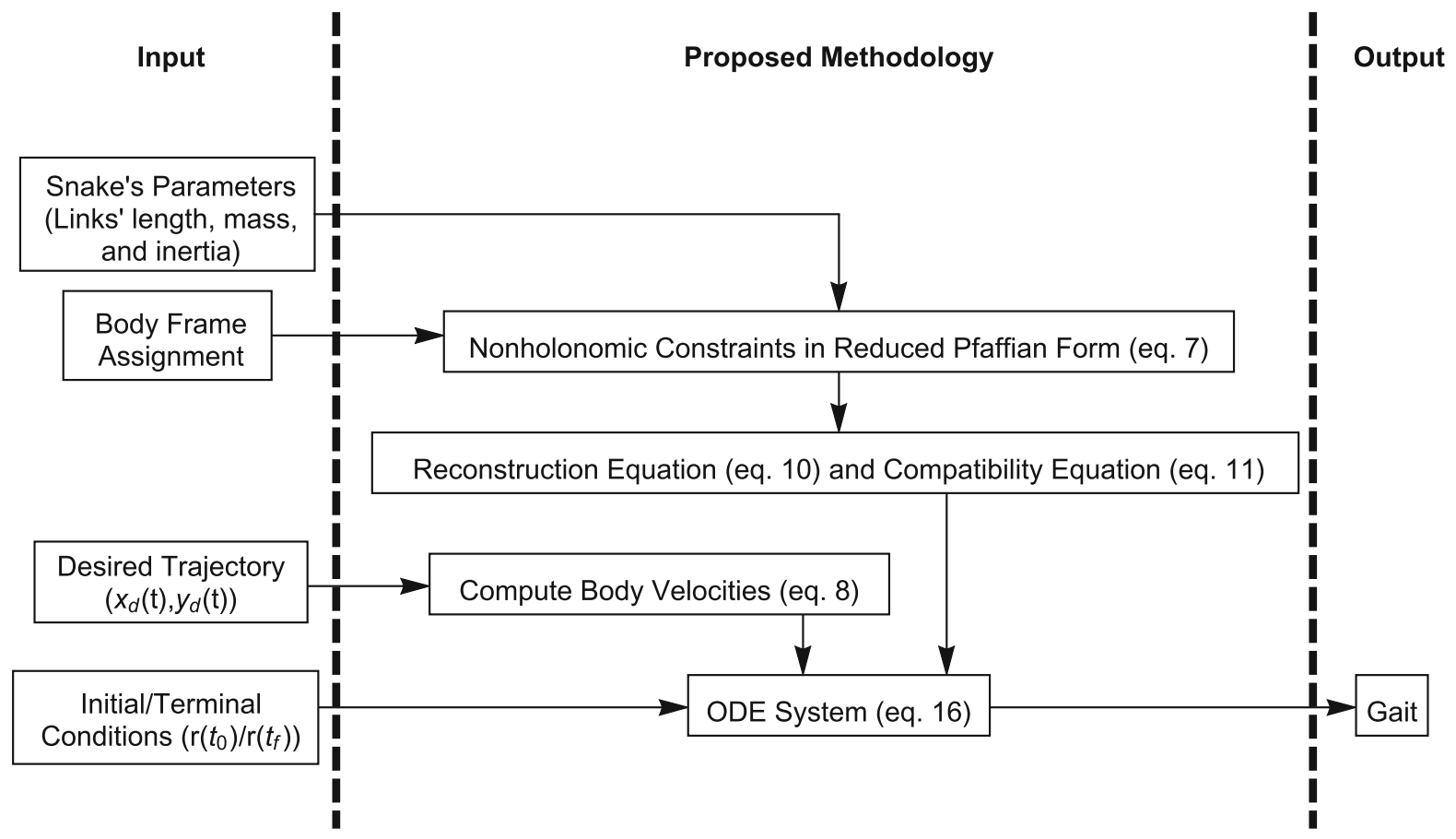

Fig. 3 Flowchart of the proposed algorithm

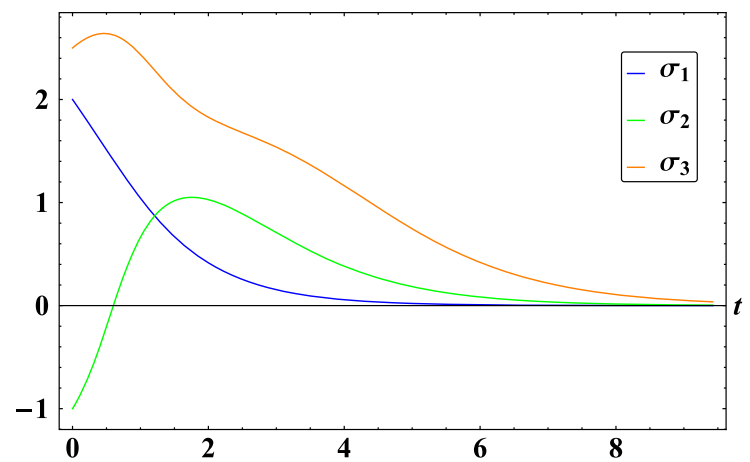

Fig. 4 The gait designed using (17) to move solely along the $x$ direction

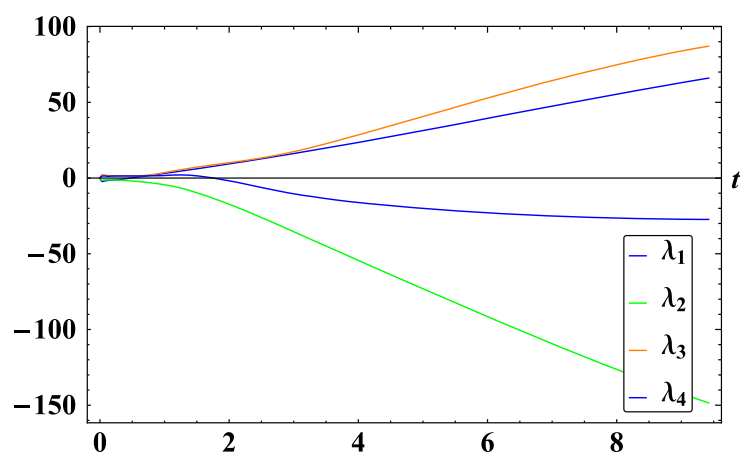

Fig. 5 The four nonholonomic forces associated with gait $r_{x}(t)$ as a function of time $t$

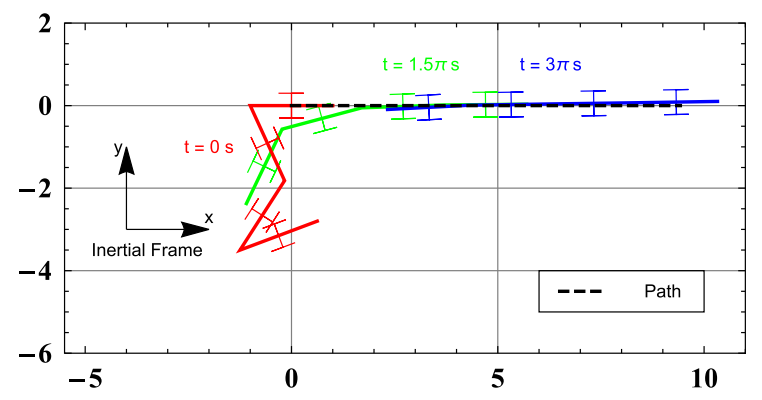

Fig. 6 The motion of the snake under the gait $r_{x}(t)$

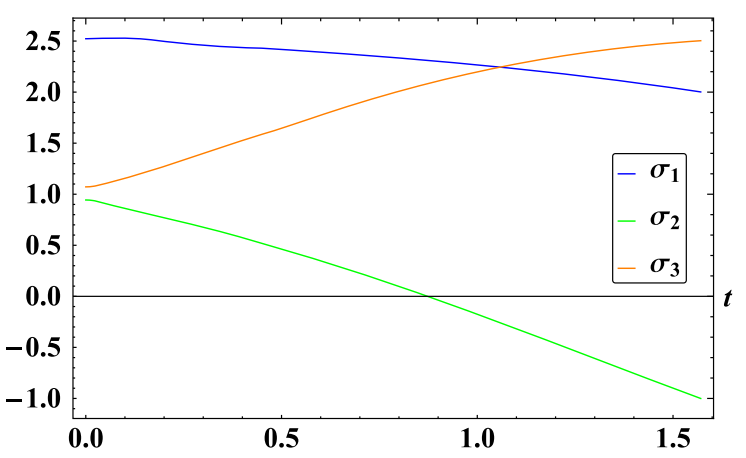

Fig. 7 Evolution of $r_{\theta}(t)$ as a function of time $t$ 
Fig. 8 The motion of the snake under the gait $r_{\theta}(t)$

Fig. 9 The four nonholonomic forces associated with the gait $r_{\theta}(t)$ as a function of time $t$

Fig. 10 The four nonholonomic forces associated with gait $r_{c}(t)$ as a function of time $t$
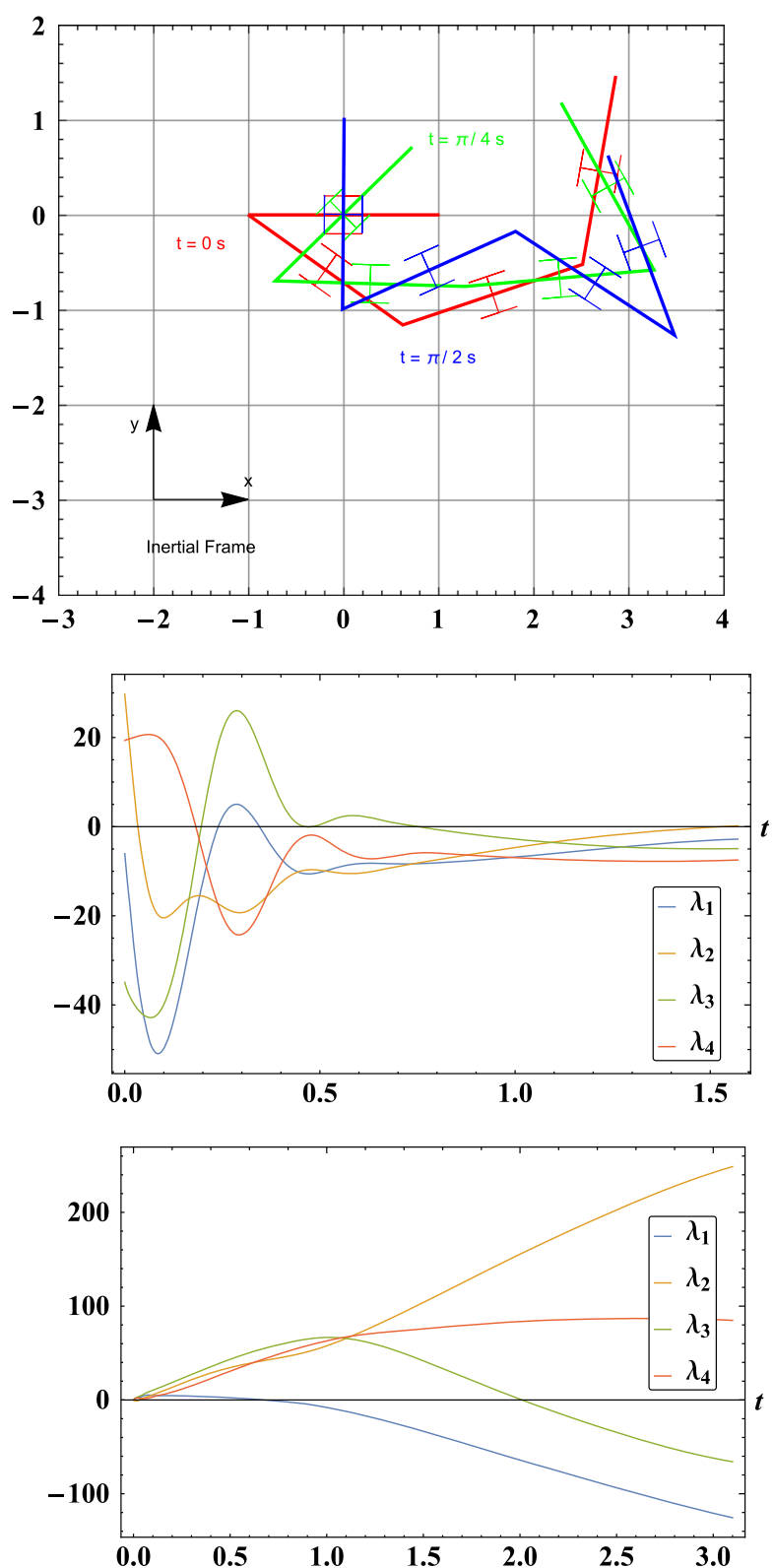


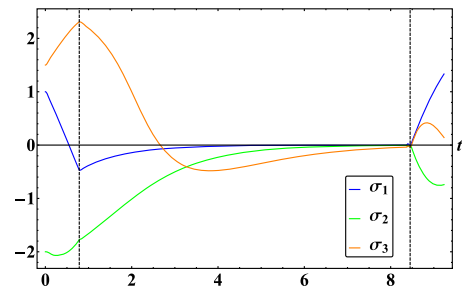

(a) Gait $r_{t m}(t)$ as function of time.

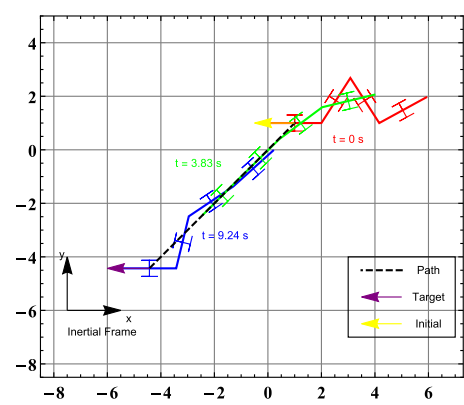

(d) Motion due to gait $r_{m t}(t)$.

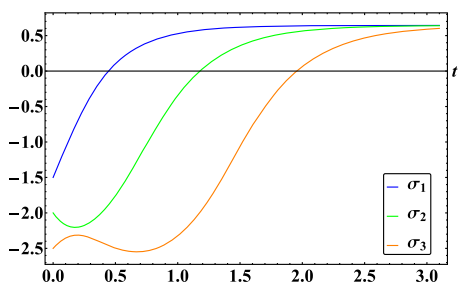

(b) Gait $r_{c}(t)$ as function of time.

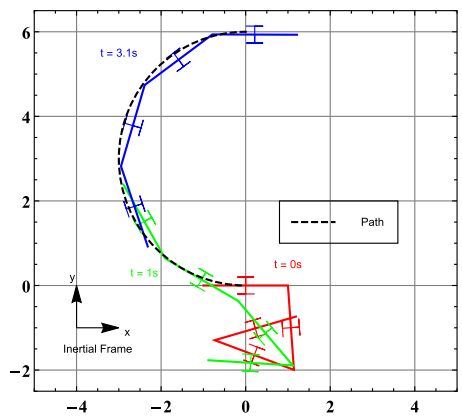

(e) Motion due to gait $r_{c}(t)$.

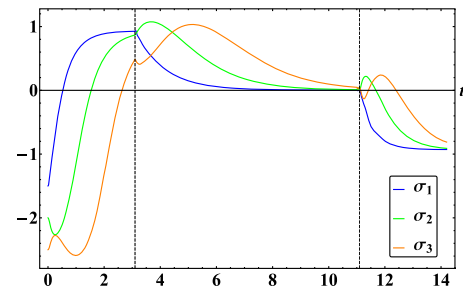

(c) Gait $r_{d}(t)$ as function of time.

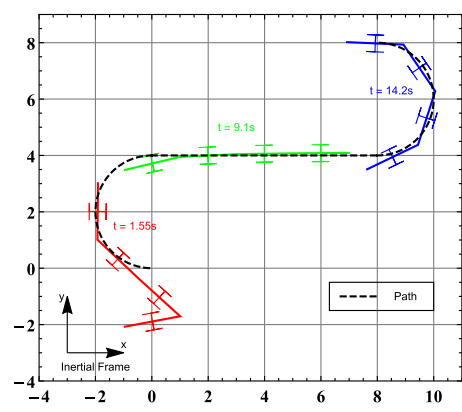

(f) Motion due to gait $r_{d}(t)$.
Fig. 11 a Evolution of $r_{t m}(t)$ components as a function of time. The dashed lines separate the gait into its three phases. b Evolution of $r_{c}(t)$ components as a function of time. $\mathbf{c}$ Evolution of $r_{d}(t)$ components as a function of time. The dashed lines separate the gait into its three phases. d Snake performing the turn-andmove motion. e Snake performing the circular motion. f Snake performing the Dubins motion

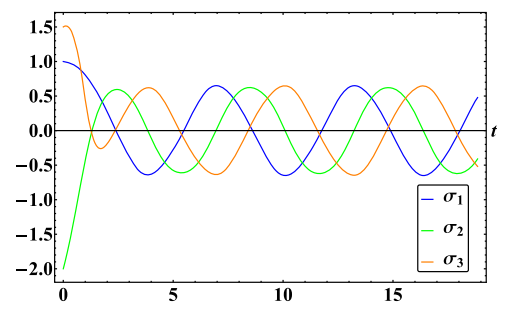

(a) Gait $r_{\sin }(t)$.

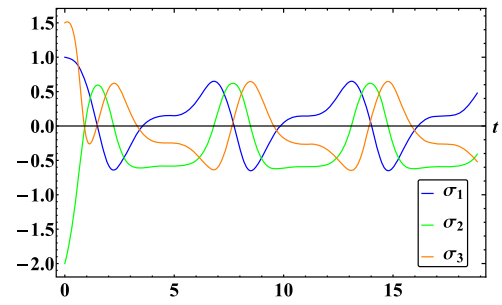

(d) Gait $r_{\text {sin-param }}(t)$.

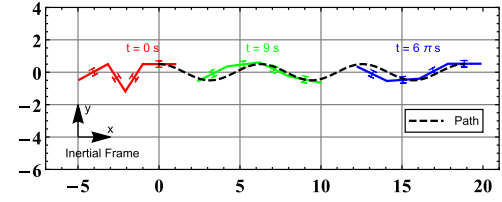

(b) Motion due to gait $r_{\sin }(t)$.

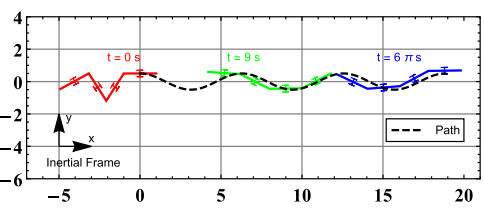

(e) Motion due to gait $r_{\sin -\text { param }}(t)$.

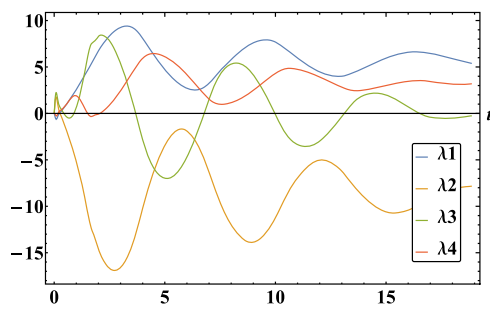

(c) Nonholonomic forces associated with gait $r_{\sin }(t)$.

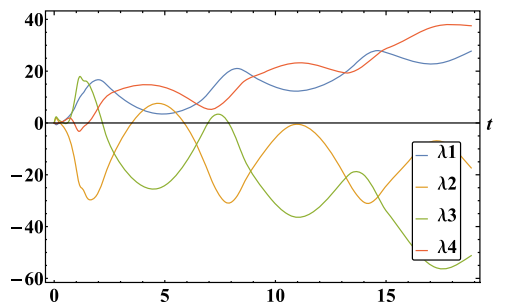

(f) Nonholonomic forces associated with gait $r_{\sin -\text { param }}(t)$.
Fig. 12 a Evolution of $r_{\sin }(t)$ components as a function of time. b Snake traversing the sinusoidal trajectory in (25). c The four nonholonomic forces associated with gait $r_{\sin }(t)$. d Evolution of $r_{\text {sin-param }}(t)$ components as a function of time. e Snake traversing the sinusoidal trajectory in (28). $\mathbf{f}$ The four nonholonomic forces associated with gait $r_{\text {sin-param }}(t)$ 


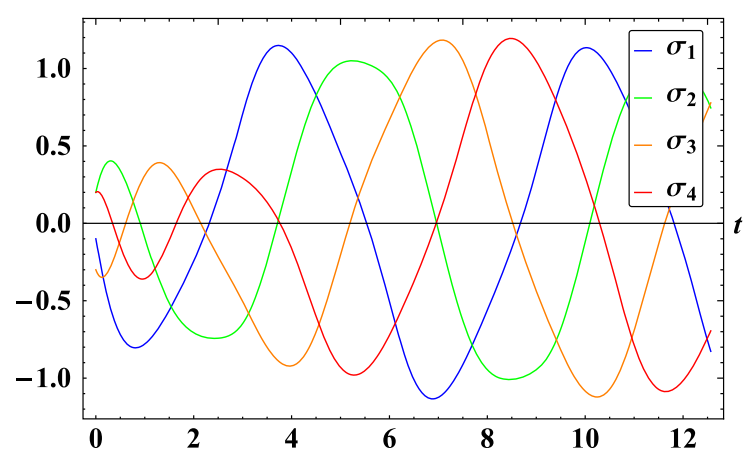

Fig. 13 The evolution of $r_{5-\operatorname{link}}(t)$ components along time $t$

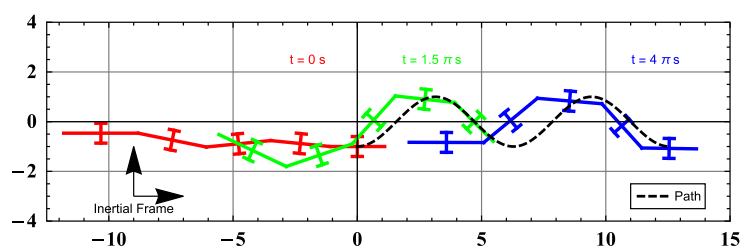

Fig. 14 The trajectory due to gait $r_{5-\operatorname{link}}(t)$

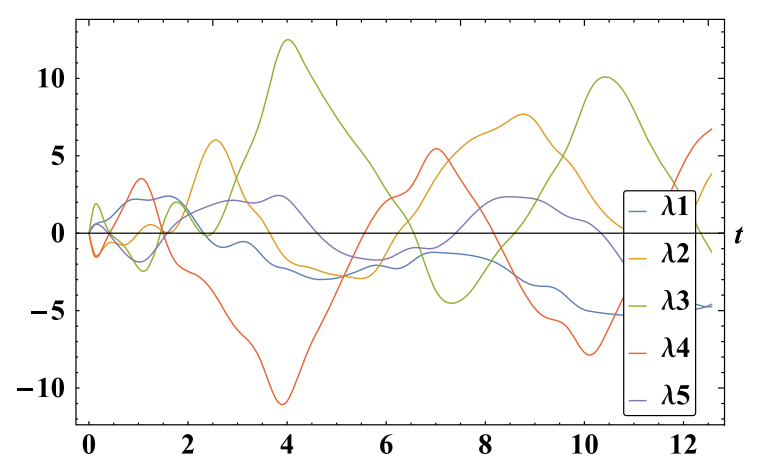

Fig. 15 The five nonholonomic forces associated with the gait $r_{5-\text { link }}(t)$ as a function of time $t$

Publisher's Note Springer Nature remains neutral with regard to jurisdictional claims in published maps and institutional affiliations. 